\title{
Research and Development of Network Virtual Laboratory Based on B/S Mode
}

\author{
Jie Ren ${ }^{1, a}$ Cuiping Pu ${ }^{1, b}$ Zongzhi Zhu ${ }^{2, c}$ Qing Zhao ${ }^{1, d}$ \\ ${ }^{1}$ Kunming University, Kunming, Yunnan, 650214,China \\ ${ }^{2}$ Zhejiang Supcon Research CO LTD, Hangzhou , 310053, China \\ arenjie-9999@163.com, bucuiping@126.com, c zhu_zz1106@163.com , d 30505860@qq.com
}

Keywords: B/S; Web; network virtual laboratory

\begin{abstract}
For colleges and universities increasing requirements in practice, but lack of experiment equipment and site problems, this paper puts forward a solution of network virtual laboratory based on B/S mode.It achieved to operate the experiments of the remote virtual laboratory directly. It has practical value and reference value.
\end{abstract}

\section{Introduction}

With the rapid development of China's higher education ${ }^{[1]}$, the increasing of the requirements of the practice teaching, lack of experimental equipment and experimental field has become a common phenomenon $^{[2]}$. This affected the development of experimental teaching and the cultivation of students' practical innovation ability in a certain extent. Distance education teaching theory, but the lack of cultivating students' practical ability, at present .Practice in distance education is very weak $^{[3]}$.In order to solve these problems, the network virtual laboratory produced with the aid of the computer network technology and relevant professional configuration software. It can not only save resources, realize the sharing of resources, avoid duplication of investment, improve the utilization of laboratory equipment, but also make learning and practice ability of students to combine theory, help to improve the teaching quality of distance education.

Compared to B/S mode and C/S mode, The opening of B/S mode is very good, the client can access through the browser, whenever and wherever possible test; $\mathrm{B} / \mathrm{S}$ mode does not need to install special client software, only need a browser, economic portable; B/S mode can be extended arbitrarily; B/S mode can make data access operation more stable, high speed the hardware level, the system interoperability and adaptability have greatly improved; B/S mode development cycle is short, easy to operate, upgrade and maintenance ${ }^{[4]}$.

\section{Hardware Architecture}

B/S (Browser/Server) mode is actually a "thin client / fat server" mode. The main application on the server, no need to install the client software client, only need it to the browser ${ }^{[5]}$. The front panel has been ported to the Web page in the B/S mode, the Web server handles related operations required. Figure 1 is the hardware architecture of network virtual laboratory based on B/S model. The Web server provides Web access service, the open experimental environment and the generatation of dynamic Webpage; provides communication service, database service and strategy service . According to the scale of the controller and the control object in network virtual laboratory ${ }^{[6]}$, we set three services deployed on one server. The actual network controller refers to the controller with network function. The actual controller and the actual control object is configured according to the needs of automation course or existing equipment. 


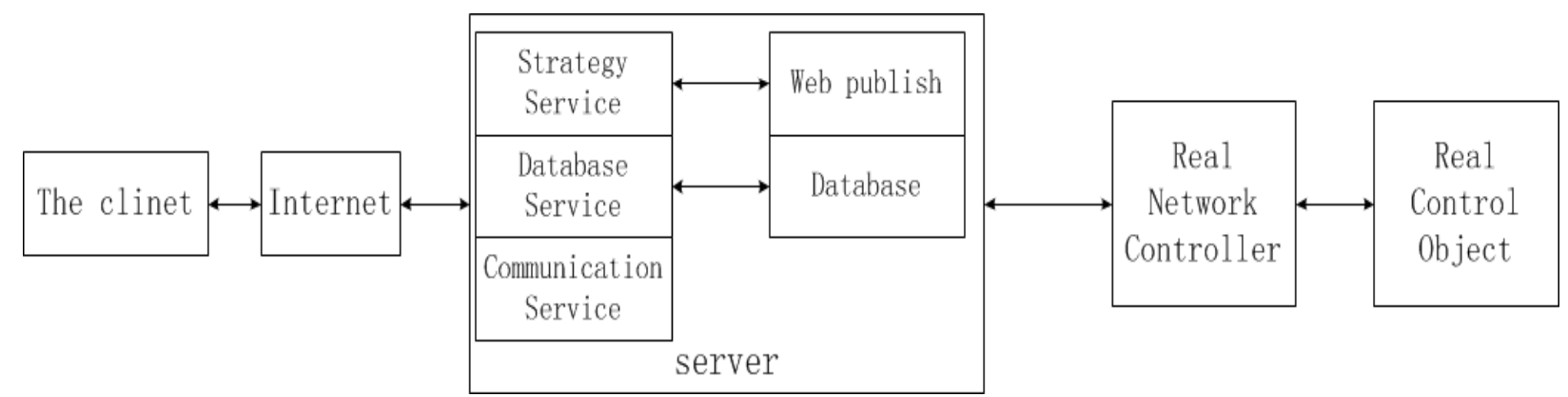

Figure 1 the hardware architecture of network virtual laboratory based on B/S model.

\section{Software Architecture}

Software development platform.The increasingly powerful function of configuration softwareprovides a platform with A powerful and flexible applicationfor the laboratory.The ForceControl configuration software is a special software acquisition and process control of the production data, with open real-time database system, can communicate with a variety of I/O equipment, and provides alarm events, provides animation, provides security protection mechanism.The EPA8000 controller is used in virtual laboratory, which provides professional control software. The control software is used to control the control object, to realize process control. Design of monitoring software of the scene.It is to find MODBUS (TCP) In the I/O Manager device of the ForceControl, The connection controller is configured, such as communication means, address configuration of all parameters. Then enter the DB Manager configuration database, complete corresponding connection the database with control object I/O. When configuration parameters, the corresponding $\mathrm{I} / \mathrm{O}$ of the corresponding area is configure, the description of the basic parameters and data connection are configured. For the bias of the $\mathrm{I} / \mathrm{O}$ point ,it is to query from the status table of the control software, to ensure that the hardware and the software match.

The establishment of the real-time database completes by creating a point parameters, connecting the I/O devices, configuring the database .It is responsible for real-time data processing, history data storage, statistical data processing, alarm information processing, data service request processing, etc ${ }^{[2]}$.

First ,the control system figure of controlling the object is drawn,by using the drawing function of ForceControl. Then,the manual button is configured, the switch oriented and the effective action are designed.etc.Finally,the animation is connectted. When the database variables change, the animation will change and move, the real-time operation state of the system can be visually seen in the animation.

Web implementation of the virtual laboratory. The Web publishing of ForceControl software supports Webserver publishing .It is a Web server that is provided by the ForceControl software, meanwhile it is a standard Web server, fully meets the standard HTTP communication protocol. ForceControl also supports IIS. The Web publishing of ForceControl is choosed, according to our actual need. First, we configure the Web server, set the IE browser, define a Web page.Then we configure the network Netserver, configure and start the Web server, start ForceControl.The Web publishing of the ForceControl can be achieved through these configuration force .

The client accesses engineering picture of the ForceControl by the Web function of the ForceControl In remote experiment process. The engineering picture display in the browser agree quite well with that is seen in view of the ForceControl operation system. Both of all the datas and animations are the same. you can also remote operation manual button to achieve the control of site control object, etc. 


\section{Conclusion}

This study realizes the direct manipulation experiment of the remote network virtual laboratory by using TCP/IP communication mode[7]. After testing, the system can realize remote operation experiment, adjust the corresponding parameter, observe the experimental results, validate control strategy and so on. This study solved the question of lacked of the experiment site and laboratory equipment and utilization rate was not high to a certain extent.it helped improve the quality of distance education practical ability to a certain extent. The experimental equipment is real, when directly controlling the remote network virtual laboratory . so a control system composed of a controller and control objects allows only one user experiment.

\section{Acknowledgements}

This work was financially supported by Applied basic research project of Yunnan Province (2013FZ098) and The scientific research fund project of Education Department of Yunnan province (2013Y257) . Data were supplied by Kunming University Library .In addition, I am grateful to the team members for their patience and good counsel. The anonymous reviewers have also contributed considerably to the publication of this paper.

\section{References}

[1] Zhao Hong , Li Zhucheng. Construction and implementation of network virtuallaboratory system based on B/S. Experim ental Technology and Management[J]. Vol.28 No .9 Sep . (2011), p. 86

[2]Sun Risheng, Sun Ziqiang.The application of ForceControl configuration software in the network virtual laboratory. Seventh industrial instrumentation and Automation Conference.(2006), p. 238

[3] Qiao Bingli, Jiang Jianfang, Xu Hui . Design and Implementation of the Remote Laboratory of PLC Control System. China Modern Educational Equipment[J]. No .1 .(2015) , p. 36

[4]Wang Shufu,Jiang Huaining,Liu Siming. Research and application of remote monitoring technology based on Web Service. Electronic technology and software engineering [J]. No .1.(2015) , p. 21

[5]ChenJingsong.ResearchonKeyTechnologiesofSettingupNetwork-basedVirtualLab.Processautom ation Instrumentation Vol.29 No.11 November (2008) , p. 43

[6]Guan Limin,Li Yang,He Yanyan. Design of remote monitoring system based on B/S model. Industrial control computer [J]. Vol.27 No .4 (2014) , p. 103

[7]Shi Mingjiang,Zhang He,He Daoqing.The design of remote process control experiment system based on the ForceControl. Micro computer information [J]. Vol.27 No .47(2011) , p. 57 